\title{
BENDING ENERGY OF HIGHLY ELASTIC MEMBRANES II
}

\author{
$\mathrm{BY}$
}

M. G. HILGERS (Dept. of Mathematics and Statistics, University of Missouri-Rolla, Rolla, MO)

AND

\section{A. C. PIPKIN ${ }^{\dagger}$ (Department of Applied Mathematics, Brown University, Providence, $R I$ )}

\begin{abstract}
The strain energy per unit area for a deformed sheet of elastic material is estimated by representing the deformation as a power series in the thickness variable. The membrane energy is the lowest-order approximation obtained in this way. Straingradient and bending energies appear in the next order of approximation. Neither the membrane energy nor the higher-order approximation satisfy the Legendre-Hadamard material stability condition if the stress is compressive in some direction, so the theories based on either of these approximations can lead to problems with no stable solution. An energy function that does satisfy the material stability conditions is obtained by omitting the strain-gradient term, provided that certain longitudinal moduli are positive. A modified form for which existence of solutions can be guaranteed is proposed.
\end{abstract}

1. Introduction. The theory of finite deformations of thin elastic membranes is based on a strain energy per unit initial area $M\left(\mathbf{r}_{. a}\right)$ that depends on the first derivatives of the deformation, $\mathbf{r}\left(x_{1}, x_{2}\right)$. A form of plate theory [1] is obtained by using an energy density of the form

$$
E=M\left(\mathbf{r}_{. a}\right)+\alpha Q\left(\mathbf{r}_{. a}, \mathbf{r}_{. a b}\right),
$$

where $\alpha$ is a positive parameter and $Q$ is a quadratic function of the second derivatives $\mathbf{r}_{. a b}$. In a previous paper [2] we have discussed the derivation of $M$ and $Q$ from the strain energy per unit initial volume $W$ for the bulk material from which the membrane is formed. We continue this discussion in the present paper and obtain a form of $Q$ that we believe to be more accurate than the form found previously.

The derivation of $M$ from $W$ is straightforward [2], but the resulting form of $M$ naturally leads to a stress-deformation relation that allows the stress to be compressive for some values of $\mathbf{r}_{. a}$. The Legendre-Hadamard material stability condition for $M$ is violated for such values of $\mathbf{r}_{. a}$; so such values can never appear in a stable solution of any problem. For this reason, it is possible to pose boundary-value problems that have no stable solution at all. The physically observed deformation in such a problem is one

Received September 15, 1993.

1991 Mathematics Subject Classification. Primary 73K10 73B05, 73G05.

$\dagger$ Deceased. 
in which the membrane is wrinkled; so it is natural to expect that existence of stable solutions might be restored by including a bending energy $\alpha Q$ that depends on the second derivatives $\mathbf{r}_{. a b}$.

For the energy (1.1), the Legendre-Hadamard condition becomes a requirement on $Q$, rather than $M$,

$$
Q\left(\mathbf{r}_{. a}, \mathbf{u} v_{a} v_{b}\right) \geq 0 \text { for all } \mathbf{u} \text { and } v_{a} .
$$

As a minimal requirement, we want (1.2) to be satisfied for all $\mathbf{r}_{. a}$. The theory is simpler in some respects if $Q$ satisfies the strong ellipticity condition,

$$
Q\left(\mathbf{r}_{. a}, \mathbf{u} v_{a} v_{b}\right)>0 \quad \text { if } \mathbf{u} \neq \mathbf{0} \text { and } \mathbf{v} \neq \mathbf{0} .
$$

A still stronger condition is positive definiteness,

$$
Q\left(\mathbf{r}_{. a}, \mathbf{r}_{. a b}\right)>0 \quad \text { unless } \mathbf{r}_{. a b}=\mathbf{0} .
$$

This is not required for stability, but if it holds, existence of solutions is relatively easy to prove.

Remarkably, the regularization achieved by adding the term $\alpha Q$ is independent of the size of $\alpha$, provided that it is positive, so the theory can be put into a well-posed form by adding an energy that is usually negligible. We conjecture that in the limit of small $\alpha$ the detailed form of $Q$ will have a negligible effect on the average deformation, although it presumably will affect the details of fine-scale wrinkling.

In spite of this conjecture, we are interested in finding the form of $Q$ that best corresponds to a given energy density $W$ for the bulk material. We have attempted to do this in an earlier paper [2]. The form of $Q$ that we obtained there has the attractive feature that it satisfies (1.2) whenever the corresponding condition for the bulk material is satisfied. However, this form disagrees with classical plate theory in the infinitesimal deformation limit, because it overestimates the bending stiffness of the material. In the present paper we obtain a form of $Q$ that does not have this defect, but its derivation is less straightforward.

Our notation is explained in Sec. 2, and in Sec. 3 we record some relations satisfied by the membrane energy function $M$. The Legendre-Hadamard condition for membranes is discussed in Sec. 4.

In Sec. 5 we express the deformation of a thin slab as a power series in the coordinate $z$ in the thickness direction, and determine the low-order coefficients in this expansion by requiring the stress to vanish on both faces of the slab. The energy per unit area $M+\alpha Q$ is then obtained as a low-order approximation to the integral of $W$ through the thickness. For materials that have reflectional symmetry in the normal direction, $Q$ separates naturally into the sum of a bending term $B$ and a strain-gradient term $S$. Then in

$$
E=(M+\alpha S)+\alpha B
$$

the strain-gradient energy $\alpha S$ appears as a modification of the stretching energy $M$, while the bending energy $\alpha B$ involves a new kind of effect.

We propose to omit the strain-gradient term $\alpha S$ and use

$$
E=M+\alpha B
$$


This is in effect what is done in classical plate theory, where there are known straingradient terms that are simply omitted (Love [3]; see also [1]). In Sec. 6 we show that this step is necessary, and not merely convenient, because the form $Q=S+B$ does not satisfy (1.2). Because of the term $\alpha S$ in (1.5), there is instability whenever the stress is compressive in some direction, just as there was when $M$ alone was used. On the other hand, the bending term $B$ does satisfy (1.2) provided that certain longitudinal moduli are nonnegative.

The form (1.6) satisfies (1.2) but not the strong ellipticity condition (1.3). In Sec. 6 we show that a slight modification of $B$ produces a form $B_{m}$ that does satisfy (1.3), at the cost of adding a fictitious strain-gradient energy that is stabilizing rather than destabilizing.

In Sec. 7 we show the forms that $M, B$, and $B_{m}$ take for deformations that involve large displacements and rotations but small strains, for isotropic materials. We verify that the forms of $M$ and $B$ found here agree with classical plate theory when the deformation is infinitesimal. The modified form $B_{m}$ is positive definite; so existence of stable solutions can be guaranteed when $B_{m}$ is used.

2. Notation. We consider a thin sheet of homogeneous elastic material that is initially bounded by the planes $x_{3}= \pm h$ in a system of Cartesian coordinates $x_{A}(A=$ $1,2,3)$. In a deformation of the sheet, the particle initially at $x_{A}$ moves to the place $\mathbf{r}^{*}\left(x_{A}\right)=r_{i}^{*}\left(x_{A}\right) \mathbf{e}_{i}$, where $\mathbf{e}_{i}(i=1,2,3)$ are orthogonal unit vectors. We usually use direct vector and dyadic notation, so that if $\mathbf{u}=u_{i} \mathbf{e}_{i}$ and $\mathbf{v}=v_{i} \mathbf{e}_{i}, \mathbf{u v}$ is the matrix with components $u_{i} v_{j}$.

The strain energy $W$ per unit initial volume is a function of the deformation gradients $\mathbf{r}_{. A}^{*}$. The derivative of $W$ with respect to $\mathbf{r}_{. A}^{*}$ is the vector

$$
\mathbf{W}_{A}=\mathbf{e}_{i} \partial W / \partial r_{i . A}^{*}
$$

and the second derivative is the matrix

$$
\mathbf{W}_{A B}=\mathbf{e}_{i} \mathbf{e}_{j} \partial^{2} W / \partial r_{i . A}^{*} \partial r_{j . B}^{*}
$$

Note that $\mathbf{W}_{B A}$ is the transpose of $\mathbf{W}_{A B}$.

$W$ depends on the deformation gradients through their inner products,

$$
\mathbf{r}_{. A}^{*} \cdot \mathbf{r}_{. B}^{*}=G_{A B}=\delta_{A B}+2 E_{A B}
$$

and we treat $W$ as a function of the strain components $E_{A B}$ defined by this relation. All functions of $E_{A B}$ are conventionally regarded as being expressed symmetrically in $E_{A B}$ and $E_{B A}$ before taking derivatives. We use the notation

$$
W_{A B}=W_{B A}=\partial W / \partial E_{A B}
$$

Then

$$
\mathbf{W}_{A}=W_{A} \mathbf{r}_{. B}^{*}
$$


Write $x_{3}=z$, so that the particle labels are $x_{a}(a=1,2)$ and $z$. Let $\mathbf{r}\left(x_{a}\right)$ be the average of $\mathbf{r}^{*}\left(x_{a}, z\right)$ through the thickness of the sheet. The membrane energy function $M$ to be defined in Sec. 3 is a function of the deformation gradients $\mathbf{r}_{. a}$, and we use notation like (2.1) and (2.2) for derivatives with respect to these variables,

$$
\mathbf{M}_{a}=\mathbf{e}_{i} \partial M / \partial r_{i . a}
$$

and

$$
\mathbf{M}_{a b}=\mathbf{e}_{i} \mathbf{e}_{j} \partial^{2} M / \partial r_{i . a} \partial r_{j . b}
$$

$M$ depends on $\mathbf{r}_{. a}$ through the inner products

$$
\mathbf{r}_{. a} \cdot \mathbf{r}_{. b}=G_{a b}=\delta_{a b}+2 E_{a b}
$$

and is thus a function of the strains $E_{a b}$ defined by this relation. We use the notation

$$
M_{a b}=\partial M / \partial E_{a b}, \quad M_{a b c d}=\partial^{2} M / \partial E_{a b} \partial E_{c d}
$$

Then

$$
\mathbf{M}_{a}=M_{a b} \mathbf{r}_{. b}
$$

and

$$
\mathbf{M}_{a b}=M_{a c b d} \mathbf{r}_{, c} \mathbf{r}_{. d}+M_{a b} \mathbf{I}
$$

where $\mathbf{I}$ is the $3 \times 3$ identity matrix.

The mean deformation $\mathbf{r}\left(x_{a}\right)$ defines a surface, and the vectors $\mathbf{r}_{a}$ are tangential to the surface. Let $\mathbf{n}$ be the unit normal vector defined by

$$
\mathbf{n}=\mathbf{N} /|\mathbf{N}|, \quad \mathbf{N}=\mathbf{r}_{.1} \times \mathbf{r}_{.2}
$$

Let $G^{a b}$ be the components of the $2 \times 2$ matrix that is inverse to $G_{a b}$, and define $\mathbf{r}^{a}$ by

$$
\mathbf{r}^{a}=G^{a b} \mathbf{r}_{. b}
$$

so that

$$
\mathbf{r}^{a} \cdot \mathbf{r}_{. c}=G^{a b} G_{b c}=\delta_{a c}
$$

The identity matrix can be expressed as

$$
\mathbf{I}=G^{c d} \mathbf{r}_{. c} \mathbf{r}_{. d}+\mathbf{n n}
$$

By using this in (2.11) we obtain

$$
\mathbf{M}_{a b}=M_{a c b d}^{*} \mathbf{r}_{. c} \mathbf{r}_{. d}+M_{a b} \mathbf{n n},
$$

where

$$
M_{a b c d}^{*}=M_{a b c d}+M_{a c} G^{b d} .
$$


3. The membrane energy function. The membrane energy function $M\left(\mathbf{r}_{, a}\right)$ corresponding to the energy density $W\left(\mathbf{r}_{, a}, \mathbf{r}_{, 3}\right)$ for the bulk material is defined as [2]

$$
M\left(\mathbf{r}_{, a}\right)=\min _{\mathbf{r}_{, 3}} 2 h W\left(\mathbf{r}_{, a}, \mathbf{r}_{, 3}\right)
$$

Let $\mathbf{r}_{, 3}=\mathbf{f}\left(\mathbf{r}_{, a}\right)$ be the minimizing value, so that

$$
M\left(\mathbf{r}_{, a}\right)=2 h W\left(\mathbf{r}_{, a}, \mathbf{f}\left(\mathbf{r}_{. a}\right)\right)
$$

Since $W$ is stationary with respect to $\mathbf{r}_{.3}$ at the minimizing value,

$$
\mathbf{W}_{3}\left(\mathbf{r}_{, a}, \mathbf{f}\left(\mathbf{r}_{, a}\right)\right)=\mathbf{0}
$$

so at this value of $\mathbf{r}_{, 3}$ there is no stress on planes $x_{3}=$ constant. With this result, the derivative of $M$ with respect to $\mathbf{r}_{, a}$ is

$$
\mathbf{M}_{a}=2 h \mathbf{W}_{a}
$$

and the second derivative with respect to $\mathbf{r}_{, a}$ and $\mathbf{r}_{, b}$ is

$$
\mathbf{M}_{a b}=2 h\left[\mathbf{W}_{a b}+\mathbf{W}_{a 3} \cdot \mathbf{F}_{b}\right]
$$

where $\mathbf{F}_{b}$ is the derivative of $\mathbf{f}$ with respect to $\mathbf{r}_{, b}$,

$$
\mathbf{F}_{b}=\mathbf{e}_{i} \mathbf{e}_{j} \partial f_{i} / \partial r_{j, b}
$$

In Sec. 5 we encounter the quantity

$$
\bar{Q}=\mathbf{f}_{a} \cdot \mathbf{W}_{a b} \cdot \mathbf{f}_{b}+2 \mathbf{f}_{, a} \cdot \mathbf{W}_{a 3} \cdot \mathbf{g}+\mathbf{g} \cdot \mathbf{W}_{33} \cdot \mathbf{g},
$$

where

$$
\mathbf{g}=\mathbf{F}_{b} \cdot \mathbf{f}_{, b} .
$$

The expression for $\bar{Q}$ can be simplified by using (3.5) and the relation

$$
\mathbf{W}_{3 a}+\mathbf{W}_{33} \cdot \mathbf{F}_{a}=\mathbf{0}
$$

which is obtained by differentiating (3.3) with respect to $\mathbf{r}_{, a}$. Using (3.8) as well, we find that

$$
Q=2 h \bar{Q}=\mathbf{f}_{, a} \cdot \mathbf{M}_{a b} \cdot \mathbf{f}_{. b} .
$$

Here $\mathbf{f}_{, a}$ is the derivative with respect to $x_{a}$,

$$
\mathbf{f}_{, a}=\mathbf{F}_{c} \cdot \mathbf{r}_{, c a} .
$$


4. Legendre-Hadamard conditions. Longitudinal moduli. Let $\mathbf{t}=t_{a} \mathbf{e}_{a}$ and $\mathbf{v}=v_{a} \mathbf{e}_{a}$ be orthogonal unit vectors, and define

$$
\mathbf{t}^{*}=\mathbf{r}_{. a} t_{a} \quad \text { and } \quad \mathbf{v}^{*}=\mathbf{r}^{a} v_{a}
$$

so that $\mathbf{t}^{*} \cdot \mathbf{v}^{*}=t_{a} v_{a}=0$, using (2.14). The $\operatorname{arc} d \mathbf{x}=\mathbf{t} d s$ is carried by the deformation onto the arc $d \mathbf{r}=\mathbf{t}^{*} d s$, and $\mathbf{v}^{*}$ is perpendicular to $d \mathbf{r}$. Now consider a family of deformation gradients $\mathbf{r}_{. a}+k \mathbf{u} v_{a}$. For all values of the parameter $k, d \mathbf{r}$ is still $\mathbf{t}^{*} d s$, but the deformation off this arc is altered. The energy density can be expanded in powers of $k$ as

$$
M\left(\mathbf{r}_{. a}+k \mathbf{u} v_{a}\right)=M\left(\mathbf{r}_{, a}\right)+k\left(\mathbf{M}_{a} v_{a}\right) \cdot \mathbf{u}+\frac{1}{2} k^{2} \mathbf{u} \cdot\left(\mathbf{M}_{a b} v_{a} v_{b}\right) \cdot \mathbf{u}+O\left(k^{3}\right) .
$$

The Legendre-Hadamard condition states that if the deformation $\mathbf{r}(\mathbf{x})$ is even weakly stable, the coefficient of $k^{2}$ here is nonnegative for all $\mathbf{u}$ and $v_{a}$ :

$$
\mathbf{u} \cdot\left(\mathbf{M}_{a b} v_{a} v_{b}\right) \cdot \mathbf{u} \geq 0
$$

By using (2.16) and choosing $\mathbf{u}=\mathbf{r}^{a} u_{a}$ we obtain

$$
M_{a b c d}^{*} v_{a} u_{b} v_{c} u_{d} \geq 0
$$

which states that certain moduli are nonnegative. By taking $\mathbf{u}=\mathbf{n}$ and using (2.16) again, we also get

$$
M_{a b} v_{a} v_{b} \geq 0
$$

This states that the normal stress on every arc is nonnegative. For, $\mathbf{M}_{a} v_{a}$ is the force per unit initial length exerted across the $\operatorname{arc} d \mathbf{r}$, and $\mathbf{v}^{*} \cdot\left(\mathbf{M}_{a} v_{a}\right)$ is proportional to its normal component. With (2.10) and (4.1),

$$
\mathbf{v}^{*} \cdot\left(\mathbf{M}_{a} v_{a}\right)=M_{a b} v_{a} v_{b} .
$$

When $\mathbf{u}=\mathbf{v}^{*}$, the perturbation $k \mathbf{v}^{*} v_{a}$ represents an extra stretching in the direction perpendicular to $d \mathbf{r}$. In this case, $u_{a}=v_{a}$ and the modulus in (4.4) is

$$
M^{\prime \prime}=M_{a b c d}^{*} v_{a} v_{b} v_{c} v_{d}
$$

In Sec. 6 we show that these longitudinal moduli are the only ones that are important in connection with the Legendre-Hadamard conditions for bending theory.

5. Bending energy and strain-gradient energy. In order to estimate the effect of bending on the stored energy of the sheet, we expand the deformation in powers of $z$ and $h$ as

$$
\mathbf{r}^{*}(\mathbf{x}, z)=\mathbf{r}(\mathbf{x})+z \mathbf{f}^{*}(\mathbf{x})+\left(\frac{1}{2} z^{2}-\frac{1}{6} h^{2}\right) \mathbf{g}^{*}(\mathbf{x})+O\left(h^{2} z\right)+O\left(z^{3}\right)
$$

where $\mathbf{x}=x_{a} \mathbf{e}_{a}$. The coefficient of $\mathbf{g}^{*}$ is written in a form that has mean zero, and the error terms are also intended to have mean zero, so that $\mathbf{r}(\mathbf{x})$ is the mean of $\mathbf{r}^{*}$ through 
the thickness. To determine $\mathbf{f}^{*}$ and $\mathbf{g}^{*}$ we require the traction on the surfaces $z= \pm h$ to vanish, so that $\mathbf{W}_{3}(\mathbf{x}, \pm h)=\mathbf{0}$. From (3.3), this is satisfied if

$$
\mathbf{r}_{.3}^{*}(\mathbf{x}, \pm h)=\mathbf{f}\left[\mathbf{r}_{, a}^{*}(\mathbf{x}, \pm h)\right]
$$

From (5.1),

$$
\mathbf{r}_{.3}^{*}(\mathbf{x}, \pm h)=\mathbf{f}^{*}(\mathbf{x}) \pm h \mathbf{g}^{*}(\mathbf{x})+O\left(h^{2}\right)
$$

and

$$
\mathbf{r}_{, a}^{*}(\mathbf{x}, \pm h)=\mathbf{r}_{. a}(\mathbf{x}) \pm h \mathbf{f}_{, a}^{*}(\mathbf{x})+O\left(h^{2}\right)
$$

Then

$$
\mathbf{f}\left(\mathbf{r}_{, a}^{*}\right)=\mathbf{f}\left(\mathbf{r}_{, a}\right) \pm h \mathbf{F}_{a} \cdot \mathbf{f}_{, a}^{*}+O\left(h^{2}\right),
$$

where $\mathbf{F}_{a}$ is defined in (3.6). Then (5.2) is satisfied with an $O\left(h^{2}\right)$ error if we take

$$
\mathbf{f}^{*}=\mathbf{f}\left(\mathbf{r}_{, a}\right) \quad \text { and } \quad \mathbf{g}^{*}=\mathbf{F}_{a} \cdot \mathbf{f}_{, a}=\mathbf{g} \text {, say. }
$$

The expansions of the deformation gradients are then

$$
\mathbf{r}_{. a}^{*}(\mathbf{x}, z)=\mathbf{r}_{, a}+z \mathbf{f}_{. a}+\left(\frac{1}{2} z^{2}-\frac{1}{6} h^{2}\right) \mathbf{g}_{, a}+O\left(h^{2} z\right)+O\left(z^{3}\right)
$$

and

$$
\mathbf{r}_{, 3}^{*}(\mathbf{x}, z)=\mathbf{f}\left(\mathbf{r}_{, a}\right)+z \mathbf{g}+O\left(h^{2}\right) \text {. }
$$

By using these expressions in $W\left(\mathbf{r}_{. a}^{*}, \mathbf{r}_{.3}^{*}\right)$ and expanding again, we obtain

$$
\begin{aligned}
W\left(\mathbf{r}_{. a}^{*}, \mathbf{r}_{.3}^{*}\right)= & W\left(\mathbf{r}_{, a}, \mathbf{f}\right)+\mathbf{W}_{a} \cdot\left[z \mathbf{f}_{, a}+\left(\frac{1}{2} z^{2}-\frac{1}{6} h^{2}\right) \mathbf{g}_{, a}\right] \\
& +\frac{1}{2} z^{2} \bar{Q}+O\left(z h^{2}\right)+O\left(z^{3}\right)+O\left(h^{4}\right)
\end{aligned}
$$

where $\bar{Q}$ is the quadratic form in $\mathbf{f}_{a}$ and $\mathbf{g}$ written out in (3.7). All derivatives of $W$ are evaluated at $\left(\mathbf{r}_{. a}, \mathbf{f}\right)$, and the term in $\mathbf{W}_{3}$ is missing from (5.9) because $\mathbf{W}_{3}\left(\mathbf{r}_{, a}, \mathbf{f}\right)=\mathbf{0}$.

By integrating (5.9) through the thickness of the sheet we obtain an expression for the energy per unit initial area,

$$
E=\int_{-h}^{h} W d z=M\left(\mathbf{r}_{, a}\right)+\frac{h^{2}}{6} Q\left(\mathbf{r}_{, a}, \mathbf{r}_{. a b}\right)+O\left(h^{4}\right)
$$

Here $M$ and $Q$ are defined by (3.2) and (3.10), respectively. Hereafter we neglect the $O\left(h^{4}\right)$ term.

We restrict attention to materials that have reflectional symmetry in the planes $x_{3}=$ constant. From (2.5), the condition $\mathbf{W}_{3}=\mathbf{0}$ that determines $\mathbf{f}$ is equivalent to $W_{3 A}=0$. For materials with reflectional symmetry this implies that $E_{3 A}=0$, so $\mathbf{f}=\phi \mathbf{n}$ where $\mathbf{n}$ is the normal vector defined by (2.12) [2]. Also, in terms of $W\left(E_{a b}, E_{3 a}, E_{33}\right)$,

$$
W_{33}\left(E_{a b}, 0, E_{33}\right)=0, \quad \text { with } \phi^{2}=1+2 E_{33} .
$$


This determines $\phi$ as a function of $E_{a b}$. Then

$$
\mathbf{f}_{. a}=\phi_{. a} \mathbf{n}+\phi \mathbf{n}_{\cdot a} .
$$

By differentiating the relations $\mathbf{n} \cdot \mathbf{n}=1$ and $\mathbf{n} \cdot \mathbf{r}_{. c}=0$ we find that

$$
\mathbf{n} \cdot \mathbf{n}_{. a}=0 \text { and } \quad \mathbf{n}_{. a} \cdot \mathbf{r}_{. c}=-\mathbf{n} \cdot \mathbf{r}_{. a c}
$$

so

$$
\mathbf{f}_{. a} \cdot \mathbf{n}=\phi_{. a} \quad \text { and } \quad \mathbf{f}_{. a} \cdot \mathbf{r}_{. c}=-\phi \mathbf{n} \cdot \mathbf{r}_{. a c} .
$$

We use these relations and the expression (2.16) for $\mathbf{M}_{a b}$ in the definition (3.10) of $Q$. This gives

$$
Q=S+B
$$

where

$$
S=\Lambda_{a b} \phi_{. l} \phi_{. b}
$$

and

$$
B=\phi^{2} M_{a b c d}^{*}\left(\mathbf{n} \cdot \mathbf{r}_{. a b}\right)\left(\mathbf{n} \cdot \mathbf{r}_{. c d}\right) .
$$

The quantities $\mathbf{n} \cdot \mathbf{r}_{. a b}$ are curvatures, and we call $B$ the bending energy. Since $\phi$ is a function of the strains $E_{a b}$, its derivative $\phi_{. a}$ depends on the strain gradients $E_{b c, a}$, and we call $S$ the strain-gradient energy.

6. Material stability conditions. As we saw in Sec. 4, the Legendre-Hadamard material stability condition is not satisfied by the membrane energy function $M\left(\mathbf{r}_{. a}\right)$ for any value of $\mathbf{r}_{\text {.a }}$ for which the stress in some direction is compressive; so such values of $\mathbf{r}_{, a}$ can never arise in a weakly stable solution of any problem. To avoid the nonexistence difficulties that this causes, we wish to replace $M$ with an energy function of the form (1.1), for which the stability condition (1.2) is a condition on $Q$ rather than $M$.

The function $Q$ obtained in Sec. 5 does not satisfy this condition for all $\mathbf{r}_{a}$. For, if we choose $\mathbf{u}$ to satisfy $\mathbf{u} \cdot \mathbf{n}=0$, then when $\mathbf{r}_{. a b}=\mathbf{u} v_{a} v_{b}$ the curvatures $\mathbf{n} \cdot \mathbf{r}_{. a b}$ are all zero and thus $Q=S$. But $\phi_{. a}$ can still be given arbitrary values by choice of $\mathbf{u}$ and $v_{a}$ [2], and from (5.16), the condition that $S$ is nonnegative for all $\phi_{, a}$ is just exactly the condition (4.5) that the stress is noncompressive. Thus the higher-order theory predicts instability whenever the stress is compressive, just as the lower-order theory did.

To obtain a higher-order theory that does satisfy (1.2), one possibility is simply to omit $S$ and use

$$
E=M\left(\mathbf{r}_{. a}\right)+\alpha B\left(\mathbf{r}_{. a}, \mathbf{r}_{. a b}\right),
$$

where $\alpha=h^{2} / 6$. This is in effect what is done in classical plate theory $[1,3]$. With $B$ given by (5.17), the Legendre-Hadamard condition becomes

$$
M_{a b c d}^{*} v_{a} v_{b} v_{c} v_{d}(\mathbf{n} \cdot \mathbf{u})^{2} \geq 0
$$

and from (4.7) we see that this is satisfied if the longitudinal modulus $M^{\prime \prime}$ is positive for every direction of stretching. 
The theory based on (6.1) has the slightly inconvenient feature that $B$ does not satisfy the strong ellipticity condition (1.3); equality holds in (6.2) when $\mathbf{n} \cdot \mathbf{u}=0$ even if $\mathbf{u} \neq \mathbf{0}$. A more convenient form of the theory is obtained by substituting

$$
B_{m}=\phi^{2} M_{a b c d}^{*} \mathbf{r}_{, a b} \cdot \mathbf{r}_{. c d}
$$

for $B$. For this form the strong ellipticity condition is

$$
M_{a b c d}^{*} v_{a} v_{b} v_{c} v_{d} \mathbf{u} \cdot \mathbf{u}>0 \quad(\mathbf{u} \neq \mathbf{0}, \mathbf{v} \neq \mathbf{0})
$$

and this is satisfied if $M^{\prime \prime}>0$.

$B_{m}$ is the sum of $B$ and a fictitious strain-gradient energy. This is seen by using (2.15) to obtain

$$
\mathbf{r}_{. a b} \cdot \mathbf{r}_{. c d}=\left(\mathbf{n} \cdot \mathbf{r}_{, a b}\right)\left(\mathbf{n} \cdot \mathbf{r}_{. c d}\right)+G^{p q}\left(\mathbf{r}_{. p} \cdot \mathbf{r}_{, a b}\right)\left(\mathbf{r}_{, q} \cdot \mathbf{r}_{. c d}\right)
$$

The factors $\mathbf{r}_{. p} \cdot \mathbf{r}_{. a b}$ in the final term can be expressed in terms of strain gradients. Consequently, $B_{m}$ still accounts for bending stiffness correctly.

7. Example. Isotropic small-strain theory. To illustrate the derivation of $M$ and $B$ from $W$, let $W$ have the form valid for infinitesimal deformations of isotropic materials,

$$
W=\frac{E}{2(1+\nu)}\left[E_{A B} E_{A B}+\frac{\nu}{1-2 \nu}\left(E_{A A}\right)^{2}\right] .
$$

Here $E$ is Young's modulus and $\nu$ is Poisson's ratio. Then

$$
W_{A B}=\frac{E}{1+\nu}\left[E_{A B}+\frac{\nu}{1-2 \nu} E_{C C} \delta_{A B}\right] .
$$

The condition (3.3), with $(2.5)$, requires that $W_{3 A}=0$. These conditions imply that

$$
E_{3 a}=0 \quad \text { and } \quad E_{33}=-\frac{\nu}{1-\nu} E_{a a} .
$$

By using these values in (7.1) and multiplying by $2 h$, we obtain

$$
M=(C / 2)\left[(1-\nu) E_{a b} E_{a b}+\nu\left(E_{a a}\right)^{2}\right],
$$

where $C$ is the notation used by Love [3] for the quantity

$$
C=2 h E /\left(1-\nu^{2}\right)
$$

To evaluate $B$ we no longer need $W$, but only $M$. We need the quantities

$$
M_{a b}=C\left[(1-\nu) E_{a b}+\nu E_{c c} \delta_{a b}\right]
$$

and

$$
M_{a b c d}=C\left[\frac{1}{2}(1-\nu)\left(\delta_{a c} \delta_{b d}+\delta_{a d} \delta_{b c}\right)+\nu \delta_{a b} \delta_{c d}\right]
$$


The value $\phi^{2}$ is

$$
\phi^{2}=1+2 E_{33}=1-\frac{2 \nu}{1-\nu} E_{a a}
$$

from (7.3). These values are to be used in (5.17).

The form (7.1) for $W$ remains valid for deformations that involve large displacements and rotations, provided that the strain components $E_{A B}$ are small enough to neglect cubic and higher-order terms. We shall simplify the expression for $B$ in the same way, by retaining only the terms of leading order. From (7.8), $\phi=1$ to leading order. From (2.17), with (7.6) and (7.7), $M_{a b c d}^{*}$ is the same as $M_{a b c d}$ to leading order. With these approximations, (5.17) gives

$$
B=C\left[(1-\nu)\left(\mathbf{n} \cdot \mathbf{r}_{. a b}\right)\left(\mathbf{n} \cdot \mathbf{r}_{. a b}\right)+\nu\left(\mathbf{n} \cdot \mathbf{r}_{. a a}\right)^{2}\right]
$$

The modified form $B_{m}$ is

$$
B_{m}=C\left[(1-\nu) \mathbf{r}_{. a b} \cdot \mathbf{r}_{. a b}+\nu \mathbf{r}_{. a a} \cdot \mathbf{r}_{. b b}\right]
$$

The Legendre-Hadamard conditions based on $B$ and $B_{m}$ are both satisfied if $C>0$, because the longitudinal modulus (4.7) is $M^{\prime \prime}=C . B$ is not strongly elliptic, much less positive definite, but in contrast, $B_{m}$ is a positive definite function of $\mathbf{r}_{a b}$ if $C>0$ and $\nu^{2}<1$. This greatly simplifies the abstract theory of existence of solutions. $B_{m}$ also leads to equilibrium equations that are simpler than those derived from $B$ [1].

We have shown in an earlier paper [1] that the energy

$$
E=M+\left(h^{2} / 6\right) B
$$

with $M$ and $B$ given by (7.4) and (7.9), reduces to the form used in classical plate theory when all displacement gradients are small. The modified form obtained by replacing $B$ with $B_{m}$ was also discussed, and it was found that the extra strain-gradient terms in $B_{m}$ give rise to boundary-layer effects at the edges of the plate.

Acknowledgment. This work was supported by a grant DMS 9301262 from the National Science Foundation. We gratefully acknowledge this support.

\section{REFERENCES}

[1] M. G. Hilgers and A. C. Pipkin, Elastic sheets with bending stiffness, Quart. J. Mech. Appl. Math. 45, $57 \cdot 75(1992)$

[2] M. G. Hilgers and A. C. Pipkin, Bending energy of highly elastic: membranes, Quart. Appl. Math. 50, 389-400 (1992)

[3] A. E. H. Love, A Treatise on the Mathematical Theory of Elasticity, 4th edition, Dover, New York. 1944 